\title{
Optimal Power Allocation for Wireless Uplink Transmissions Using Successive Interference Cancellation
}

\author{
Liaoyuan Wu, Yamei Wang, Jianghong Han, Wenqiang Chen, and Lusheng Wang \\ School of Computer and Information, Hefei University of Technology \\ 193 Tunxi Road, 230009, Hefei, Anhui, China \\ [e-mail: wanglusheng@hfut.edu.cn] \\ *Corresponding author: Lusheng Wang
}

Received November 4, 2015; revised February 14, 2016; accepted March 26, 2016;

published May 31, 2016

\begin{abstract}
Successive interference cancellation (SIC) is considered to be a promising technique to mitigate multi-user interference and achieve concurrent uplink transmissions, but the optimal power allocation (PA) issue for SIC users is not well addressed. In this article, we focus on the optimization of the PA ratio of users on an SIC channel and analytically obtain the optimal PA ratio with regard to the signal-to-interference-plus-noise ratio (SINR) threshold for successful demodulation and the sustainable demodulation error rate. Then, we design an efficient resource allocation (RA) scheme using the obtained optimal PA ratio. Finally, we compare the proposal with the near-optimum RA obtained by a simulated annealing search and the RA scheme with random PA. Simulation results show that our proposal achieves a performance close to the near-optimum and much higher performance than the random scheme in terms of total utility and Jain's fairness index. To demonstrate the applicability of our proposal, we also simulate the proposal in various network paradigms, including wireless local area network, body area network, and vehicular ad hoc network.
\end{abstract}

Keywords: Successive interference cancellation, power allocation, resource allocation, uplink transmission

This research was supported by the National Natural Science Foundation of China under Grant No. 61501160, also in part supported by International Science \& Technology Cooperation Program of China under Grant No. 2014DFB10060. 


\section{Introduction}

To satisfy various forthcoming high-throughput and real-time applications of the mobile Internet, new multiple access techniques for wireless networking have become an important reseach direction. However, traditional multiple access schemes require users to access a single channel at different time slots. Otherwise, multiple access interference (MAI) is introduced and severely degrades the performance of the whole network [1]-[3]. Interference management techniques could be used to overcome this problem [4]. Typical ideas include interference avoidance (IA) and interference cancellation (IC) [5]. IA is achieved by certain deterministic scheduling or random access protocols. An example for deterministic scheduling is time division multiple access (TDMA); while an example for random access is carrier sensing multiple access with collision avoidance (CSMA/CA).

All of the IA schemes try to avoid overlapped usage of the same time slots by multiple users, and their upperbound performance is not nearly enough to support near-future applications of the mobile Internet [6]. With the enhancement of the signal processing capability of the physical layer, IC schemes have become quite attractive in recent years. These schemes can be categorized into parallel interference cancellation (PIC) and successive interference cancellation (SIC). SIC overcomes PIC's extremely high demands on hardware and has become a practical and efficient IC technique [7]. The idea is to simultaneously demodulate multiple signals from multiple users based on a certain iteration process [8], which can be divided into three steps. First, the received signals from multiple users are ranked from the strongest to the weakest. Second, the strongest signal can be demodulated and subtracted from the mixed received signal as long as its signal-to-interference-plus-noise ratio (SINR) is larger than a certain threshold. Third, the second strongest signal can be demodulated, and the iteration continues until all of the signals are demodulated successfully or until any signal's demodulation fails due to low SINR.

J. G. Andrews [2] provided a survey of various IC schemes and compared their methods for implementation, indicating the practicability of the SIC technique. Research on SIC has mainly focused on the computational complexity and the enhancement of successful signal detection [9]-[12]. However, existing studies have rarely focused on the power allocation (PA) to increase SIC schemes' performance on fading channels. [12] claimed that the performances of SIC on the additive white Gaussian noise (AWGN) channel and the Rayleigh fading channel could be substantially different. [9] introduced a PA scheme, but instead of PA on continuous power values, it only considered two discrete power values indicating high and low transmission power settings.

In this article, we focus on the optimization of the PA ratio of multiple users on an SIC channel and the corresponding resource allocation (RA) scheme. The contribution of this study is twofold. On the one hand, we theoretically determine the optimal PA ratio for the Rayleigh fading channel with regard to the sustainable demodulation error rate and the required SINR for successful demodulation. On the other hand, we propose a fast RA scheme using the optimal PA ratio, and compare it with random PA and the near-optimum obtained by a simulated annealing search to demonstrate its high performance.

The remainder of this article is organized as follows. In Section 2, we summarize the related works. In Section 3, we describe the system model for SIC under the Rayleigh fading channel. In Section 4, we theoretically determine the optimal PA ratio for SIC. In Section 5, 
various RA schemes with different PA settings are described. In Section 6, we demonstrate the performance of the scheme with optimal PA ratio in various network paradigms and various RA schemes with different PA settings. Finally, in Section 7, we draw the conclusions.

\section{Related Work}

The idea of SIC was first raised by T. Cover [13], and recent studies discussed its applications in various types of networks. M. Wildemeersch et al. [14] proposed an SIC-based multi-layer analytical model for heterogeneous cellular networks, which focused on the wireless channel features of randomly distributed users and access points (APs). S. Sasipriya and C. S. Ravichandran [15] suggested the integration of PIC and SIC to mitigate multi-user interference in CDMA networks. In addition, several studies that worked on SIC-based schemes in multi-hop wireless networks are available [5][16][17]. C. Jiang et al. [5] established a mixed cross-layer integer programming model and introduced a joint scheme using SIC and interference exploitation together for the improvement of the network performance. Based on that study, Y. Shi et al. [16] introduced a rapid iteration-based solution, while Q. Long et al. [17] further considered the impact of transmission rate controlling on network performance. Most of the studies on the SIC technique have focused on the uplink, but D. Hu and S. Mao [10] integrated super coding to extend SIC to downlink multicast transmissions.

There are also many studies on the performance evaluation and implementation of the SIC technique. R. Yim et al. [9] focused on the design of an SIC-based receiver using local channel information and received signal strength indication (RSSI), but the impact of unideal channel or power control on the performance of the SIC-based receiver was not discussed. R. Fa and R. C. de Lamare [11] proposed a multi-branch SIC and analysed the impact of channel modelling on the network performance. L. Yang [12] proposed multi-user diversity-aided multi-stage minimum mean-square error (MMSE) multi-user detection and analysed its performance on both AWGN and Rayleigh fading channels. A. Zanella and M. Zorzi [18] analysed the usage of SIC in heterogeneous networks. Poisson point process (PPP) was used to model the user distribution, and the impact of pathloss and Rayleigh fading on the network performance was analysed. X. Zhang and M. Haenggi [19] derived the SIC-based successful demodulation probability and the normalized throughput and analysed its performance in a scenario with pathloss and Rayleigh fading. However, the above two studies did not consider proactive PA, so they did not achieve near-optimal SIC performance like the scheme proposed in this article. R. Yim et al. [9] introduced a proactive PA method for SIC but only considered two discrete power values.

In summation, it is important to determine the optimal PA ratio of different users in an SIC channel under Rayleigh fading. An optimal PA ratio could benefit the design of an optimal SIC scheme by allocating as many users as possible in each channel, hence improving the spectral efficiency. However, none of the above existing studies provided any optimal or near-optimal solution to this issue. Therefore, our work proposes a near-optimal solution by combining theoretical and simulative results. Moreover, our scheme establishes proactive PA values based on the obtained near-optimal PA ratio, so it does not require feedback and reaction for PA adjustment. 


\section{System Model}

Let us consider a scenario where an AP provides $N$ channels for $\boldsymbol{M}$ users denoted by $\left\{U E_{m} \mid m=1,2, \ldots, M\right\}$, as shown in Fig. 1. SIC allows multiple users to be assigned to the same channel but to transmit with powers of different magnitudes. Taking Fig. $\mathbf{1}$ as an example, channels 1 and 4 contain two users each, while channels 2 and 3 contain three users each.

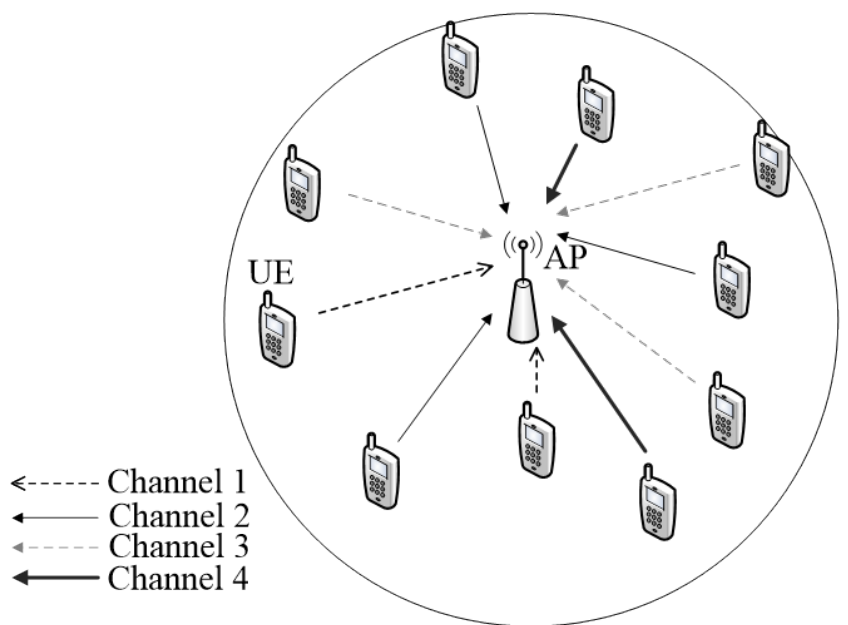

Fig. 1. Wireless uplink transmission scenario.

The uplink transmitting power of $U E_{m}$ and the pathloss from $U E_{m}$ to the AP are denoted by $P_{T}^{m}$ and $P L_{m}$, respectively. Thus, the average receiving power at the AP can be written as [20]

$$
\overline{P_{R}^{m}}=P_{T}^{m} / P L_{m} .
$$

Under Rayleigh fading, the instantaneous receiving power at the AP from $U E_{m}$ on channel $\boldsymbol{n}$ is written as

$$
P_{R}^{m n}=\overline{P_{R}^{m}} \cdot R a y l_{n}^{m},
$$

where $R a y l_{n}^{m}$ represents the Rayleigh fading of $U E_{m}$ on channel $n$ [21].

SIC ranks the users on the same channel by their instantaneous receiving power and starts the demodulation procedure from the largest to the smallest one-by-one. To simplify the notations in the system model and the derivations later, we consider that the users are ranked based on their instantaneous receiving power ascendingly. When we demodulate the signal of $U E_{m}$, signals of $U E_{l}, l=m+1, \ldots, M$ should be successfully demodulated and eliminated from the received mixed signal, which should not interfere with the demodulation of $U E_{m}$ at all. Therefore, the instantaneous SINR for the uplink transmission to the AP from $U E_{m}$ can be written as 


$$
\operatorname{SINR}(m, n)=\frac{P_{R}^{m n} \cdot \eta_{m n}}{\sum_{i=1}^{\mathrm{m}-1} P_{R}^{i n} \cdot \eta_{i n}+N_{0}}
$$

where $N_{0}$ represents the variance of AWGN, and

$$
\eta_{\text {in }}=\left\{\begin{array}{l}
0, \text { for } U E_{i} \text { not using channel } n, \\
1, \text { for } U E_{i} \text { using channel } n .
\end{array}\right.
$$

To demodulate successfully the signal of $U E_{m}$, the SINR indicated by (3) should be no smaller than $\theta(\theta>1)$ [19]. Thus, the spectral efficiency of $U E_{m}$ can be written as

$$
S E^{m n}=\left\{\begin{array}{cc}
\log _{2}[1+\operatorname{SINR}(m, n)] & \operatorname{SINR}(m, n) \geq \theta \\
0 & \operatorname{SINR}(m, n)<\theta \text { or } S E^{(m+1) n}=0
\end{array}\right.
$$

Because different users have different application requirements, terminal properties, and user preferences, spectral efficiency may not reflect the actual utility directly. Based on a number of representative studies on RA [22][23], we use a sigmoidal utility function to represent the quality of service (QoS) of each user. Because the pathloss values of various users can be measured in reality, we can adjust the transmitting power to obtain the desired average receiving power so that the instantaneous receiving power involving Rayleigh fading can be set to a reasonable range to guarantee the number of users satisfying SIC conditions, hence maximizing the sum of utilities. Therefore, the optimization for the multi-user multi-channel RA problem in this article can be written as

$$
\begin{gathered}
\max \quad F=\sum_{n=1}^{N} \sum_{m=1}^{M} s\left(S E^{m n}\right) \\
\text { s.t. } P_{\min } \leq P_{R}^{m n} \leq P_{\max } \text {, and } \\
\eta_{m n}=0,1, \forall m \in\{1, \ldots, M\}, n \in\{1, \ldots N\}
\end{gathered}
$$

where $s(x)=(x / b)^{a} /\left[1+(x / b)^{a}\right]$ is the sigmoidal utility function, $b$ is its mid value, and $a$ is the variance ratio. $P_{\min }$ and $P_{\max }$ represent the minimum and the maximum receiving power for certain wireless networks, which are usually defined in their standards.

\section{Optimal PA Ratio}

SIC demodulates signals from the largest to the smallest. Each demodulated signal can be removed from the mixed received signal so that the smaller signals can be further demodulated without interference from larger ones. For each user, we assume that its signal can be successfully demodulated as long as the SINR is no less than $\theta$. Because $N_{0}$ is a very small value compared with the interference, it can be ignored for simplification. Therefore, to guarantee successful demodulation of the signal from $U E_{m}$ as well as the minimum gap between the receiving power strengths of different users, we have

$$
P_{R}^{m n} / \sum_{i=1}^{m-1} P_{R}^{i n}=\theta, m=2,3, \ldots, L
$$

where $L$ is the number of users in channel $n$. To satisfy the condition in (7), if we define the 
average receiving power of $U E_{1}$ as unit 1, the average receiving power of $U E_{2}$ should be $\theta$ if we ignore the impact of AWGN and Rayleigh fading. Then because the additive power strength for these two users is $(1+\theta)$, the average receiving power of $U E_{3}$ should be $(1+\theta) \theta$ to guarantee the condition in (7), and so on. To sum up, we obtain the average receiving power strengths of $L$ users in a single channel with the following ratio

$$
\left\{1, \theta,(1+\theta) \theta,(1+\theta)^{2} \theta,(1+\theta)^{3} \theta, \ldots,(1+\theta)^{L-2} \theta\right\} .
$$

Due to Rayleigh fading, the instantaneous receiving power obeys Rayleigh distribution. When the average receiving power obeys the ratio in (8), their instantaneous receiving power values do not, which makes some users' SINR values lower than $\theta$ and causes failure of demodulation. To overcome this problem, we bring in a series of anti-fading factors, i.e., $\delta_{m-1}, m=2, \ldots, L$, so the average receiving power values of $L$ users in a single channel become

$$
P_{R}^{m n} / \sum_{i=1}^{m-1} P_{R}^{i n}=\theta+\delta_{m-1}, m=2,3, \ldots, L .
$$

Therefore, the average receiving power of $U E_{2}$ is adjusted as $\theta+\delta_{1}$, that of $U E_{3}$ is adjusted as $\left(1+\theta+\delta_{1}\right)\left(\theta+\delta_{2}\right)$, and so on. Hence, the ratio in (8) becomes

$$
\begin{gathered}
\left\{1, \theta+\delta_{1},\left(1+\theta+\delta_{1}\right)\left(1+\theta+\delta_{2}\right),\left(1+\theta+\delta_{1}\right)\left(1+\theta+\delta_{2}\right)\left(\theta+\delta_{3}\right),\right. \\
\cdots \cdots \\
\left.\left(1+\theta+\delta_{1}\right)\left(1+\theta+\delta_{2}\right) \cdots\left(1+\theta+\delta_{L-2}\right)\left(\theta+\delta_{L-1}\right)\right\} .
\end{gathered}
$$

We can see that by fixing $\theta$, the probability for successful demodulation is determined by this series of anti-fading factors. If these factors are too small, it is not effective against fading, resulting in low capacity. By contrast, if these factors are too large, the containable number of users per channel is limited, resulting in low resource utilization. Therefore, it is a vital issue to find the optimal series of $\delta_{m-1}, m=2,3, \ldots, L$, which is the purpose of the following subsections.

\subsection{Calculation of $\delta_{1}$}

For the first user,

$$
P_{R}^{1 n}=\overline{P_{R}^{1}} \cdot \text { Rayl }_{n}^{1}=\text { Rayl }_{n}^{1} .
$$

Hence,

$$
\operatorname{SINR}(1, n)=P_{R}^{1 n} / N_{0}=\operatorname{Rayl}_{n}^{1} / N_{0} .
$$

$N_{0}$ is a very small value compared with the receiving power Rayl ${ }_{n}^{1}$. For example, we assume that $N_{0}=-105 \mathrm{dBm}, \theta=1.1$, and Rayl ${ }_{n}^{1}$ obeys Rayleigh distribution with its mode equal to 1 . Rayl $I_{n}^{1}>N_{0} \theta$ corresponds to successful demodulation, indicating that the demodulation error rate is calculated by $P_{E}=\int_{-\infty}^{N_{0} \theta} \operatorname{Rayl}_{n}^{1} d x \approx 6.05 \times 10^{-22}$. Therefore, we conclude that $U E_{1}$ could almost always be successfully demodulated.

For $U E_{2}$,

$$
P_{R}^{2 n}=\left(\theta+\delta_{1}\right) \text { Rayl }_{n}^{2}
$$


Hence,

$$
\operatorname{SINR}(2, n)=\frac{P_{R}^{2 n}}{P_{R}^{1 n}+N_{0}} \approx \frac{\left(\theta+\delta_{1}\right) \operatorname{Rayl}_{n}^{2}}{\operatorname{Rayl}_{n}^{1}} .
$$

where Rayl $l_{n}^{1}$ and Rayl ${ }_{n}^{2}$ obey identical and independent distribution (i.i.d.), so the probability density function (PDF) of $\operatorname{SINR}(2, n)$ can be obtained (by Appendix A) as

$$
f_{2}(s)=\frac{2 s\left(\theta+\delta_{1}\right)^{2}}{\left[s^{3}+\left(\theta+\delta_{1}\right)^{2}\right]^{2}} .
$$

The sustainable demodulation error rate of $U E_{2}$ is denoted as $\alpha$, and it corresponds to the probability of $\operatorname{SINR}(2, n)<\theta$, i.e.,

$$
\alpha=\int_{-\infty}^{\theta} f_{2}(s) d s .
$$

Based on the derivation in Appendix B, we obtain

$$
\delta_{1}=(\sqrt{1 / \alpha-1}-1) \theta .
$$

\subsection{Calculation of $\delta_{2}$}

For the third user,

$$
\operatorname{SINR}(3, n)=\frac{\overline{P_{R}^{3}} \cdot \text { Rayl }_{n}^{3}}{\overline{P_{R}^{1}} \cdot \text { Rayl }_{n}^{1}+\overline{P_{R}^{2}} \cdot \text { Rayl }_{n}^{2}+N_{0}} \approx \frac{\left(1+\theta+\delta_{1}\right)\left(\theta+\delta_{2}\right) \cdot \text { Rayl }_{n}^{3}}{\operatorname{Rayl}_{n}^{1}+\left(\theta+\delta_{1}\right) \cdot \text { Rayl }_{n}^{2}} .
$$

Based on the derivation in Appendix C, we obtain the PDF of $g=\left(\theta+\delta_{1}\right) x+y$ as

$$
f_{g}(g)=\frac{g}{(c+1)^{2} \sigma^{2}} e^{-\frac{g^{2}}{2 \sigma^{2}}}+\frac{c g}{(c+1)^{2} \sigma^{2}} e^{-\frac{g^{2}}{2 c \sigma^{2}}}+\left[\frac{g^{2}}{(c+1)^{2} \sigma^{3}}-\frac{1}{(c+1) \sigma}\right] e^{-\frac{g^{2}}{2(c+1) \sigma^{2}}} * p(g)
$$

where $c=\left(\theta+\delta_{1}\right)^{2}$ and

$$
p(g)=\sqrt{\frac{\pi c}{2+2 c}}\{\operatorname{erf}[c \cdot q(g)]+\operatorname{erf}[q(g)]\}, \text { with } q(g)=\frac{g}{\sigma} \sqrt{\frac{1}{2 c(c+1)}} .
$$

Because there are erf functions in $f_{g}(g)$, it is impossible to analytically obtain $f_{3}(s)$, the $\operatorname{PDF}$ of $\operatorname{SINR}(3, n)$, in the same way as $f_{2}(s)$, so a curve fitting method is used. We generate $R a y l_{n}^{1}, R_{a y l}^{2}$, and $R a y l_{n}^{3}$, obeying i.i.d. Rayleigh distribution, and $\operatorname{SINR}(3, n)$ is obtained based on (18). Then, we use the ksdensity function in Matlab to statistically obtain the PDF histogram. Finally, curve fitting is performed to obtain the polynomial function using least-square criterion, denoted by $f_{3}{ }^{\prime}(s)$, which could be quite close to $f_{3}(s)$ when the degree of this polynomial function is large. Similar to (16), we have

$$
\int_{-\infty}^{\theta} f_{3}^{\prime}(s) d s=\alpha .
$$

We show the relationship between $\delta_{2}$ and the two parameters $\alpha$ and $\theta$ as the curves in Fig. 2. Along with the increment of $\theta, \delta_{2}$ increases linearly. At the same time, $\delta_{2}$ decreases along with the increment of $\alpha$. These features are the same as the relationship between $\delta_{1}$ and 
these two parameters, which inspires us to draw $y=(\sqrt{1 / \alpha-1}-1) x$ (the markers in Fig. 2) and compare it with the curves obtained by curve fitting above. We can see that the curves and the markers perfectly match with each other for various $\alpha$ and $\theta$ values. Therefore, we conclude that
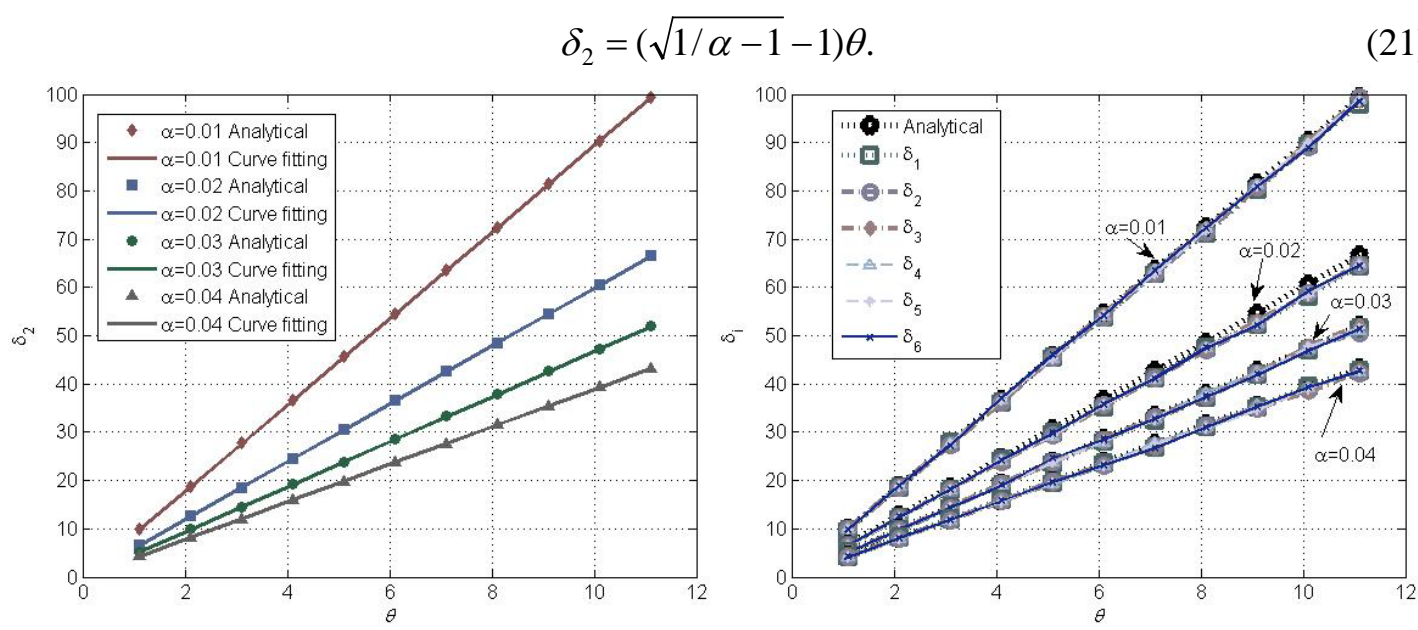

Fig. 2. Analytical and curve fitting results for $\delta_{2}$. Fig. 3. $\delta_{i}(i=1,2, \ldots 6)$ with regard to $\alpha$ and $\theta$.

\subsection{Calculation of $\delta_{i}, i=3,4,5,6$}

For $\delta_{i}, i=3,4,5,6$, using the same method as Subsection 4.2, we draw the curve fitting results and $y=(\sqrt{1 / \alpha-1}-1) x$ in Fig. 3. A similar conclusion is obtained, i.e.,

$$
\delta_{i}=(\sqrt{1 / \alpha-1}-1) \theta, i=3,4,5,6 .
$$

Because each wireless networking protocol defines its maximum and minimum achievable/allowed receiving power strengths, each channel in SIC could generally contain at most six or seven users, so $\delta_{6}$ (corresponding to the 7 th user) is complete enough to demonstrate the feature of the series of $\delta_{i}$. Therefore, we conclude that, all of the $\delta_{i}$ should obey (22) for the SIC issue.

\section{RA Schemes With Different PA Settings}

\subsection{Simulated annealing search toward the system optimality}

In this subsection, we propose an idea for searching for a near-optimal PA and channel assignment solution for the SIC-based multi-user multi-channel RA problem. For each $\theta$, the corresponding $\delta_{i}$ can be calculated based on (22), so the maximum number of containable users per channel can be obtained by combining $\delta_{i}$ and the maximum receiving power $P_{\max }$. For the sake of fairness, we try to equally allocate channels to the users during each assignment period, so a permutation of all of the users will be used to assign users to channels. If some remaining spaces in some channels are still available, a few randomly selected users will fill in these blanks, guaranteeing that no user was assigned twice in these remaining spaces. In this way, the initial state of such a combinatorial optimization issue is formed. 
Because the instantaneous receiving power involves not only pathloss but also Rayleigh fading, many users in the initial state may not be successfully demodulated. Therefore, a searching procedure is required to gradually converge to the optimal solution. In this article, we choose the simulated annealing algorithm for this purpose. The detailed procedure is shown by the flowchart in Fig. 4. First, the maximum number of containable users per channel is calculated, and the initial assignment solution is generated. Then, the total cost of this initial solution is calculated, and the simulated annealing procedure is started. During simulated annealing, we change one user's assignment each time and calculate the new solution's total utility. If the total utility increases, the new solution is accepted as the best and further search continues from there. If the total utility decreases, there is a probability of obeying the Metropolis rule to accept it and to continue further search from it. Finally, when the searching time expires, the algorithm is stopped, and a near-optimal solution is obtained. Note that the utility function for calculating the total utility is performed based on the SIC technique. Once the signal of a user cannot be demodulated, the signals with lower power strengths will have to be discarded without demodulation, so their utilities should be set to zeros.

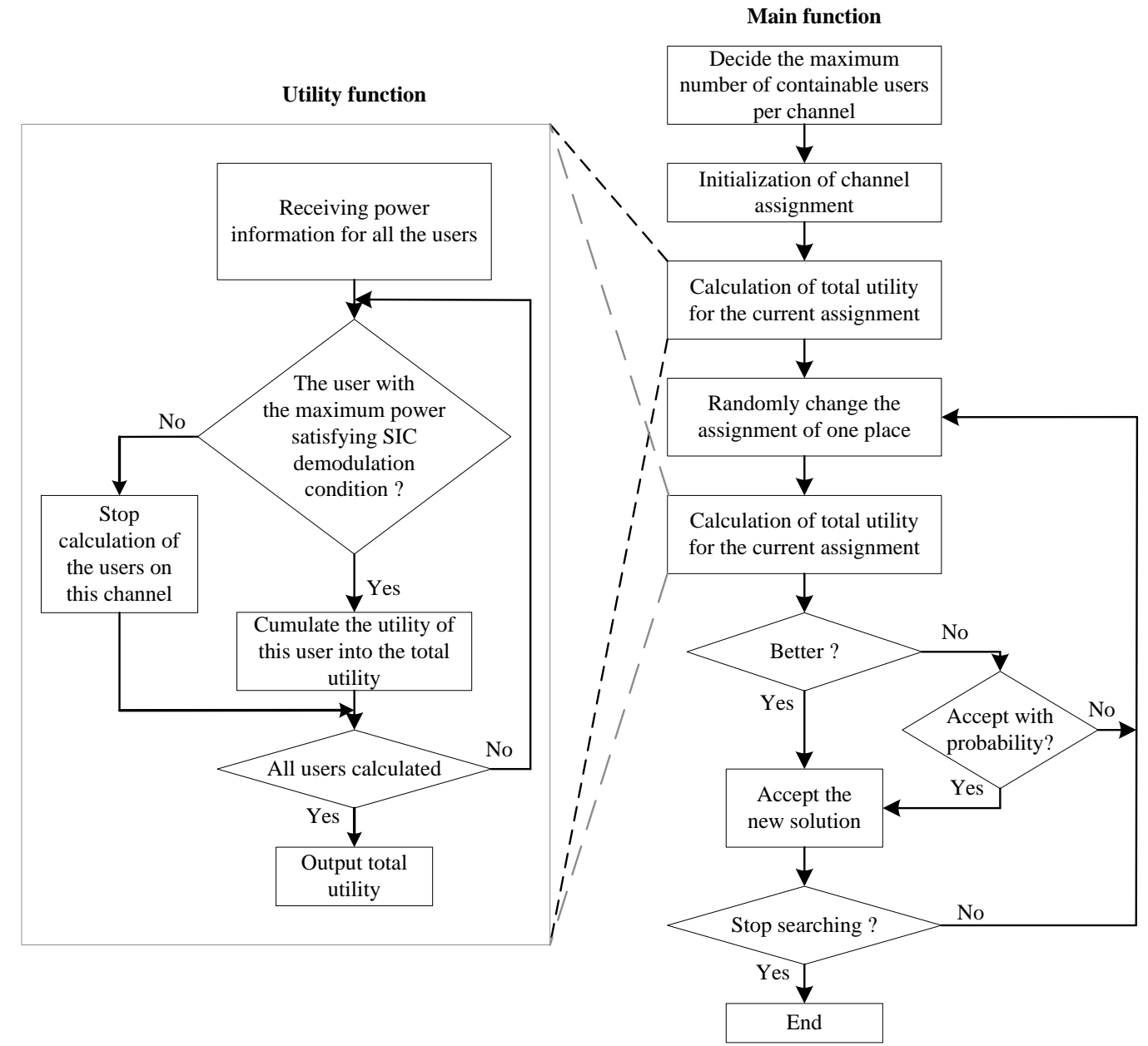

Fig. 4. Flowchart for searching toward the system optimality using simulated annealing. 


\subsection{Fast RA with optimal PA ratio}

The above algorithm can find a near-optimal solution but takes quite a long time, so it could be used for near-optimum searching but not for practical usage in an SIC-based communication system. In this subsection, we propose a practical and efficient RA scheme to quickly achieve a good solution for PA and channel assignment for the SIC technique.

First, the average power strengths of the places in each channel are set by obeying (10) and (22). Second, the users are assigned to the places in multiple channels randomly. Once a user is assigned to a certain place in a channel, its average receiving power should be adjusted to obey the power ratio indicated by (10) and (22), as shown in Fig. 5. Finally, if some places in some channels are still available because the number of users may be less than the total number of available places, a set of randomly selected users will be assigned to the remaining places. In this way, the optimal power ratio indicated by (10) guarantees successful demodulation of most users, so the network performance could be relatively high.

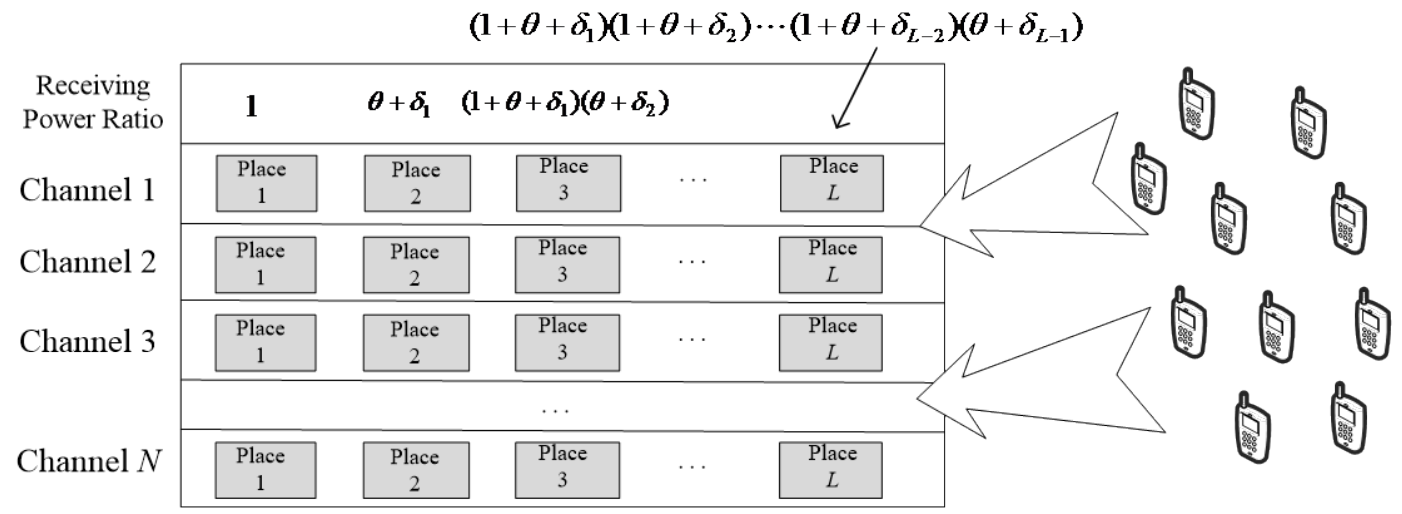

Fig. 5. RA with optimal PA ratio, i.e., fixed power ratio for places in each channel.

\subsection{RA with random PA}

To compare with the above-proposed optimal PA scheme, we also consider a scheme with random PA, i.e., without a predefined power ratio like (10). In this case, channel assignment should not be completely random. Otherwise, the probability to successfully demodulate the users in each channel would be too low. Therefore, we consider a simple heuristic channel adjustment idea as follows.

The users with small average receiving power strengths should be assigned to the left-most places in the above scheme. Meanwhile, the users with high average receiving power strengths should be assigned to the right-most places. For each channel, the selected users between the one with the maximum power and the one with the minimum power obey almost arithmetic sequence. In this way, we try the best to demodulate successfully the signals of users as many as possible. 


\section{Numerical Results}

\subsection{Application of our proposal on WLAN, BAN, and VAN}

In this subsection, we apply the proposed PA scheme in Section 4 for wireless local area network (WLAN), body area network (BAN), and vehicular ad hoc network (VAN). WLAN is widely used everywhere in the world. One of its latest standard is [24]. BAN is a new paradigm of wireless sensor network techniques and has quite wide applications in various domains [25]. One of its latest standard is IEEE 802.15.6 for on-body and around-body short-distance wireless communications [26]. VAN is the vehicular communication network for intelligent transportation system and one of its latest standards is IEEE 802.11p [27]. We refer to these standards for the typical power setting parameters of these networks and obtain Table 1 below.

Table 1. Parameters for various wireless network protocols

\begin{tabular}{|c|c|c|c|}
\hline Parameter & WLAN 802.11af & BAN 802.15.6 & VAN 802.11p \\
\hline \hline Receiver sensibility $P_{\min }$ & $-88 \mathrm{dBm}$ & $-95 \mathrm{dBm}$ & $-82 \mathrm{dBm}$ \\
\hline Maximum receiving power $P_{\max }$ & $-20 \mathrm{dBm}$ & $0 \mathrm{dBm}$ & $-20 \mathrm{dBm}$ \\
\hline$P_{\max } / P_{\min }$ & $10^{6.8}$ & $10^{9.5}$ & $10^{6.2}$ \\
\hline
\end{tabular}

In the following simulations, the ratio of average receiving powers is set as (10), and $\delta_{i}$ are set as (22). Different network standards define the maximum and the minimum receiving power strengths, given by $P_{\max }$ and $P_{\min }$ below. To simplify the expression, let the mininum receiving power of each channel being unit 1 and the maximum receiving power and the number of containable users $L$ obey $(1+\theta+\delta)^{L-2}(\theta+\delta) \leq P_{\max } / P_{\min }$. Therefore, the maximum number of containable users per channel can be obtained, which will guide the design of the channel assignment. Because the minimum receiving power is set to unit 1 as mentioned above, all of the receiving powers are equal to or larger than this magnitude. Therefore, we set AWGN to 0.00001, which is a value small enough to be unsubstantial in the calculation of the SINR. Moreover, the scale parameter of Rayleigh distribution $\sigma$ is set to 1 , the mid value $b$ of the sigmoidal utility function is 3 , and its variance ratio $a$ is 2 . To obtain smooth curves, we perform 500,000 rounds of simulations and take the average to draw the curves in the following figures. We analyse the impact of $\alpha$ and $\theta$ on the network performance, i.e., the number of successfully demodulated users and the total utility.

Fig. 6 shows the impact of $\alpha$ and $\theta$ on the total utility in WLAN. For any given $\theta$, with the increment of $\alpha$, the trend of the total utility is to first increase dramatically and then dcrease slowly. For $\theta=1.1$, the total utility is maximized at $\alpha=0.021$, so we obtain the optimal $\delta=6.4106$ based on (22). Similarly, for $\theta=2,3$, the optimal $\delta=8.8265$ and 15.0093, respectively. The optimal $\delta$ obtained in this way will be used in the following simulations. 


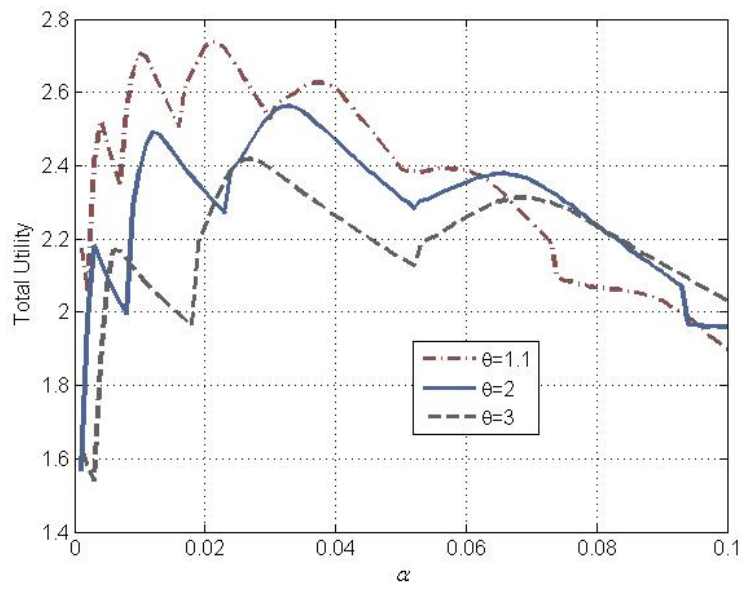

Fig. 6. Total utility vs. $\alpha$ and $\theta$ for WLAN.

Fig. 7 shows the optimal $\delta$ for the maximization of the containable users with different $\alpha$ and $\theta$ in WLAN, BAN, and VAN. As explained before, each channel has a maximum receiving power $P_{\max }$. For a given $\theta$, the optimal $\delta$ should correspond to the user with the maximum receiving power setting to a value close to $P_{\max }$. When $\theta$ increases, the overall trend of the optimal $\delta$ is to increase, while it decreases slightly at some parts of $\theta$ and then jumps to a much higher value. The reason is revealed by (10) and explained as follows: to guarantee the same number of containable users and to avoid $P_{R}^{L n}$ exceeding $P_{\max }$, when $\theta$ increases, $\delta$ must correspondingly decrease. However, when $\delta$ cannot be decreased anymore, the maximum number of containable users has to decrease by 1 , corresponding to the jumps of the optimal $\delta$ to a higher value at some points.

Fig. 8 shows the maximum number of containable users for different $\delta$ values. Consider an ideal case where only the pathloss is used to model the channel, the optimal $\delta$ is obtained as explained above, so the maximum number of containable users for this ideal case in Fig. 8 also jumps at corresponding $\theta$ values as in Fig. 7. When Rayleigh fading is taken into account, it is quite possible that some users cannot be successfully demodulated. Therefore, we need to check one-by-one from the strongest to the weakest receiving power to determine the number of users this channel could support, hence obtaining the three smooth curves with Rayleigh fading in Fig. 8. We can see that with the increment of $\theta$, the maximum number of containable users gradually decreases. Meanwhile, by comparing the three networks, the BAN parameters lead to a large number of containable users. 


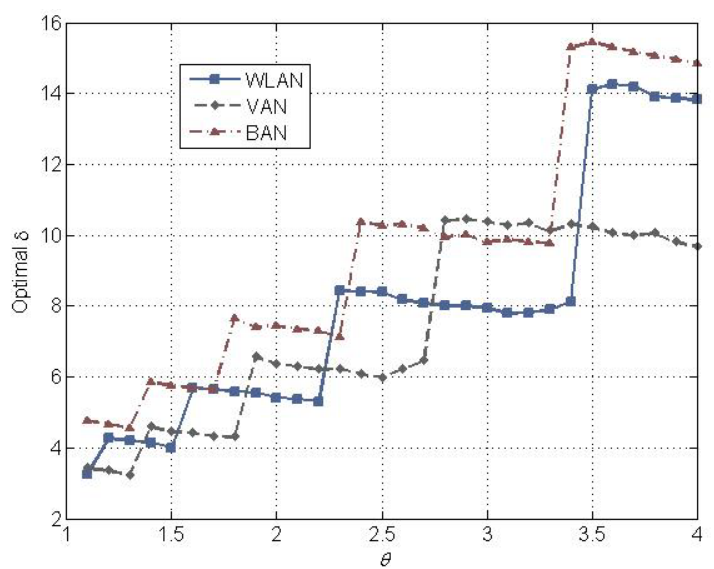

Fig. 7. Optimal $\delta$ for the maximum containable users.

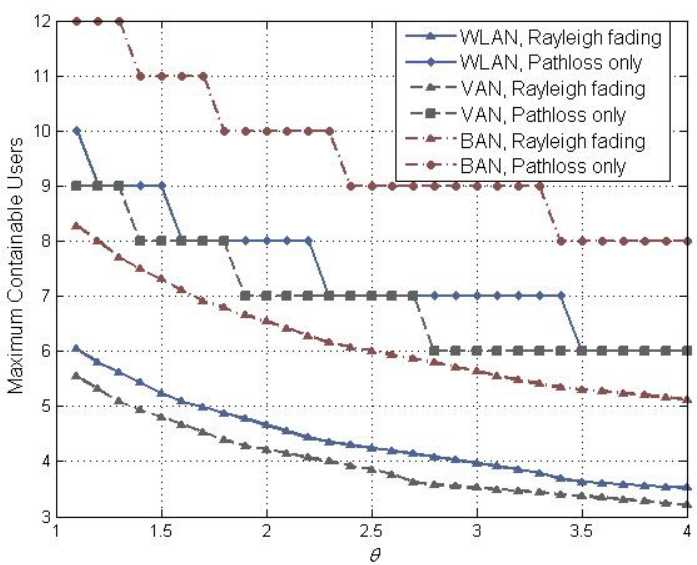

Fig. 8. Maximum containable users.

For Fig. 9 and Fig. 10, we change the objective function into the total utility of all of the successfully demodulated users and show the changes of the optimal $\delta$ values and the total utility, respectively. The overall trends of the curves in these figures are the same as those in Fig. 7 and Fig. 8. Meanwhile, BAN parameters still lead to better network performance than the other two network settings. With Rayleigh fading, many users may not be able to be demodulated, so the total utility decreases dramatically. Moreover, we should notice that the optimal $\delta$ values in Fig. 9 are larger than those in Fig. 7 on average. Larger $\delta$ values lead to fewer containable users, indicating that with the total utility as its objective, the maximum number of containable users shown in Fig. 8 is not necessarily achieved.

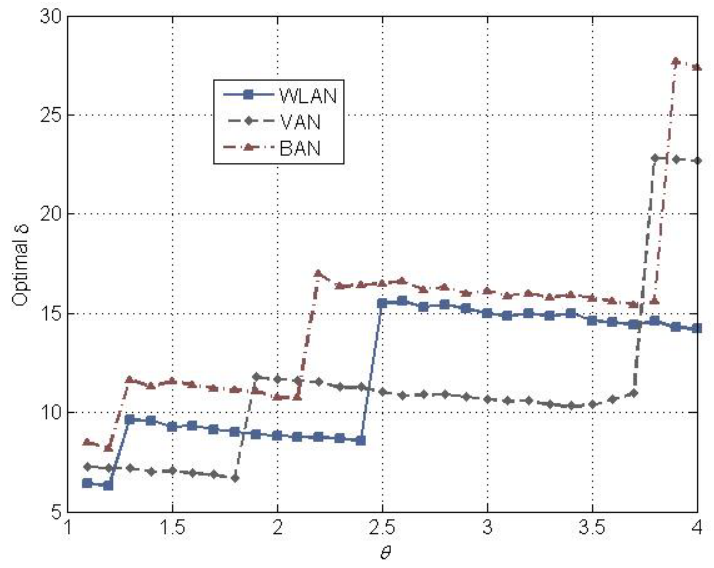

Fig. 9. Optimal $\delta$ for the maximum total utility.

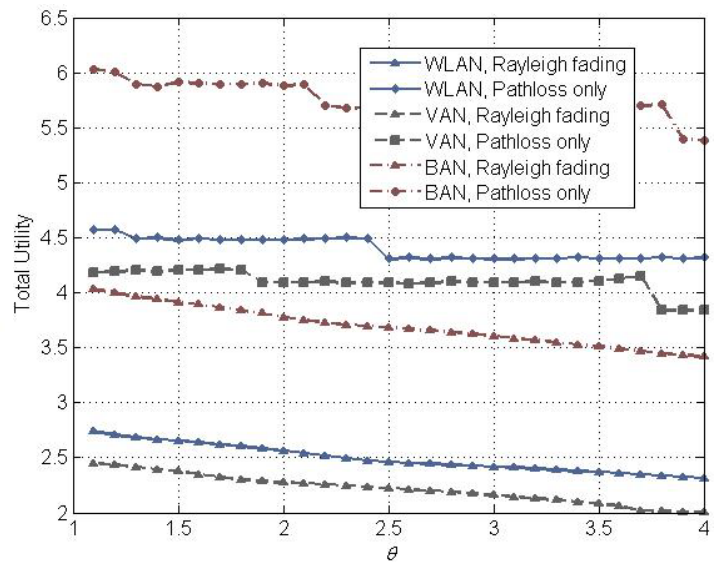

Fig. 10. Maximum total utility.

\subsection{Performance evaluation of RA schemes with different PA settings}

In this subsection, we compare the performance of the proposed optimal PA scheme with both the random and near-optimal solution obtained by simulated annealing search in the WLAN scenario. The three schemes have been described in Section 5. Simulation parameters are set as in the previous subsection. For simulated annealing, the initial temperature is set to 100 , the temperature decreasing rate is 0.99 , and the stopping temperature is 0.01 . 
Fig. 11 shows the total utilities of different schemes. We can see that the proposed efficient RA scheme with optimal PA achieves a close performance to the near-optimum obtained by simulated annealing search and much higher than the scheme with random PA. Fig. 12 compares the Jain's fairness values of these schemes. The trends of these curves are the same as those of the curves in Fig. 11, indicating the high performance of the proposed scheme.

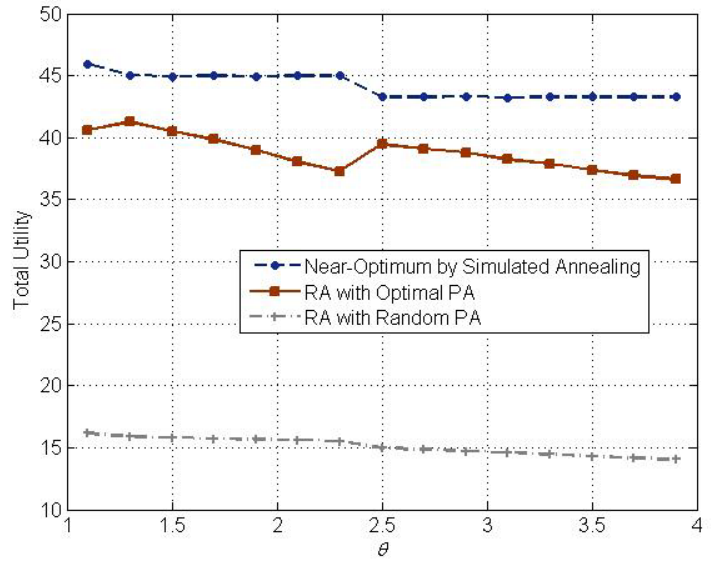

Fig. 11. Comparison of the total utility.

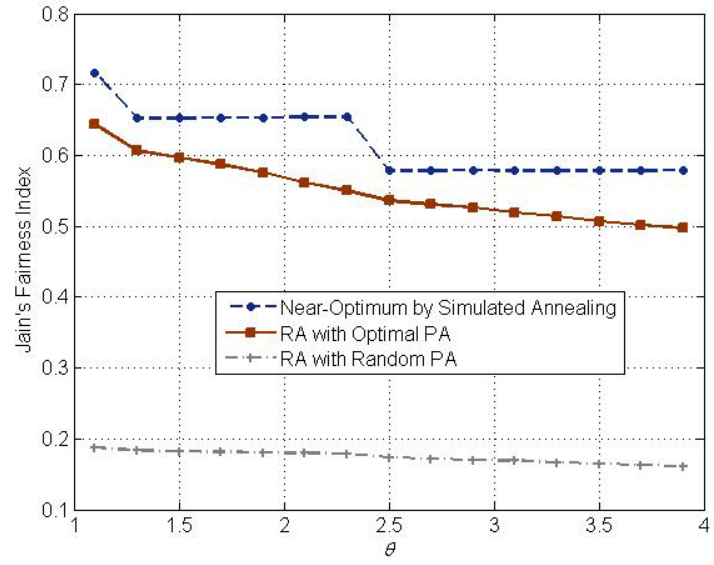

Fig. 12. Comparison of the Jain's fairness index.

Note that, along with the increment of $\theta$, all of the curves tend to decrease. According to (5), one reason is that the probability of successful demodulation decreases. The other reason is the increment of $\delta$ along with the increase of $\theta$, which may decrease the maximum number of containable users per channel. The change of the maximum number of containable users per channel results in jumps at some points for the near-optimal curves. Note that there is a small augmentation of the curve for the proposed scheme around $\theta=2.5$. The reason for this augmentation is that when the maximum number of containable users decreases to 1 at each jump point, the probability of successful demodulation increases, which may sometimes lead to a small performance augmentation, such as $\theta=2.5$ in Fig. 11.

\section{Conclusion}

SIC technique has been widely studied, but its PA in fading channels has not been solved. In this article, we focused on the optimization of the allocated power for SIC-based RA schemes. The optimal PA ratio of a set of users using a single SIC channel was analytically obtained. An RA scheme using this optimal PA ratio was proposed. Simulations showed that this scheme achieved a performance close to the near-optimum obtained by simulated annealing search and much higher than the scheme with random PA. Our future work is to extend the proposed scheme to other scenarios, such as Rician fading channels and log-normal shadowing. 


\section{Appendix A}

For simplicity, $x$ and $y$ are used to represent Rayl $_{n}^{1}$ and Rayl ${ }_{n}^{2}$, respectively, and (14) can be written as $s=\left(\theta+\delta_{1}\right) x / y$, so the joint PDF of $x$ and $y$ is $f(x, y)=\frac{x y}{\sigma^{4}} e^{-\frac{x^{2}+y^{2}}{2 \sigma^{2}}}$. Given

$$
\left\{\begin{array}{l}
x=\frac{y s}{\theta+\delta_{1}} \\
y=y
\end{array}\right.
$$

the Jacobian determinants can be calculated as

$$
J=\left|\begin{array}{ll}
\frac{\partial x}{\partial y} & \frac{\partial x}{\partial s} \\
\frac{\partial y}{\partial y} & \frac{\partial y}{\partial s}
\end{array}\right|=\left|\begin{array}{cc}
\frac{s}{\theta+\delta_{1}} & \frac{y}{\theta+\delta_{1}} \\
1 & 0
\end{array}\right|=-\frac{y}{\theta+\delta_{1}} .
$$

Then, the joint PDF of $y$ and $s$ can be written as

$$
f(y, s)=f(x, y)|J|=\frac{|y|}{\theta+\delta_{1}} f\left(\frac{y s}{\theta+\delta_{1}}, y\right)=\frac{|y|^{3} s}{\left(\theta+\delta_{1}\right)^{2} \sigma^{4}} e^{-y^{2} \frac{\left(\frac{s}{\theta+\delta_{1}}\right)^{2}+1}{2 \sigma^{2}}} .
$$

Therefore, the PDF of $s$ can be calculated by

$$
\begin{gathered}
f_{2}(s)=\int_{-\infty}^{+\infty} f(y, s) d y=\int_{-\infty}^{+\infty} \frac{|y|^{3} s}{\left(\theta+\delta_{1}\right)^{2} \sigma^{4}} e^{-y^{2} \frac{s^{2}+\left(\theta+\delta_{1}\right)^{2}}{2 \sigma^{2}\left(\theta+\delta_{1}\right)^{2}}} d y \\
=\frac{s}{\left(\theta+\delta_{1}\right)^{2} \sigma^{4}} \int_{0}^{+\infty} y^{3} e^{-y^{2} \frac{s^{2}+\left(\theta+\delta_{1}\right)^{2}}{2 \sigma^{2}\left(\theta+\delta_{1}\right)^{2}}} d y=\frac{s}{s^{2}+\left(\theta+\delta_{1}\right)^{2}} \frac{1}{\sigma^{2}} \int_{0}^{+\infty}-y^{2} d\left(e^{-y^{2} \frac{s^{2}+\left(\theta+\delta_{1}\right)^{2}}{2 \sigma^{2}\left(\theta+\delta_{1}\right)^{2}}}\right) .
\end{gathered}
$$

Based on the integration by parts $\int u d v=u v-\int v d u$, we have

$$
\begin{gathered}
f_{2}(s)=\left.\frac{s}{s^{2}+\left(\theta+\delta_{1}\right)^{2}} \frac{1}{\sigma^{2}}\left(-y^{2}\right) e^{-y^{2} \frac{s^{2}+\left(\theta+\delta_{1}\right)^{2}}{2 \sigma^{2}\left(\theta+\delta_{1}\right)^{2}}}\right|_{0} ^{+\infty}-\frac{s}{s^{2}+\left(\theta+\delta_{1}\right)^{2}} \frac{1}{\sigma^{2}} \int_{0}^{+\infty} e^{-y^{2} \frac{s^{2}+\left(\theta+\delta_{1}\right)^{2}}{2 \sigma^{2}\left(\theta+\delta_{1}\right)^{2}}} d\left(-y^{2}\right) \\
=-\frac{2 s\left(\theta+\delta_{1}\right)^{2}}{\left[s^{2}+\left(\theta+\delta_{1}\right)^{2}\right]^{2}}\left[\int_{0}^{+\infty} e^{-y^{2} \frac{s^{2}+\left(\theta+\delta_{1}\right)^{2}}{2 \sigma^{2}\left(\theta+\delta_{1}\right)^{2}}} d\left(-y^{2} \frac{s^{2}+\left(\theta+\delta_{1}\right)^{2}}{2 \sigma^{2}\left(\theta+\delta_{1}\right)^{2}}\right)\right] \\
=-\left.\frac{2 s\left(\theta+\delta_{1}\right)^{2}}{\left[s^{2}+\left(\theta+\delta_{1}\right)^{2}\right]^{2}} e^{-y^{2} \frac{s^{2}+\left(\theta+\delta_{1}\right)^{2}}{2 \sigma^{2}\left(\theta+\delta_{1}\right)^{2}}}\right|_{0} ^{+\infty}=\frac{2 s\left(\theta+\delta_{1}\right)^{2}}{\left[s^{2}+\left(\theta+\delta_{1}\right)^{2}\right]^{2}}
\end{gathered}
$$




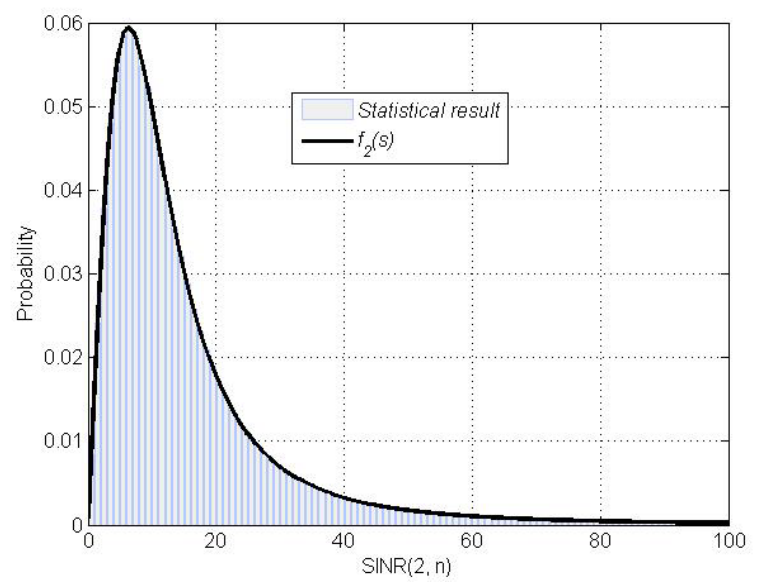

Fig. 13. Verification of the PDF derived in Appendix A.

Let $\theta=1.1$ and $\delta_{1}=9.85$, we draw the curve of $f_{2}(s)$ in Fig. 13. By generating random numbers obeying the Rayleigh distribution, and by taking their division as in (14), we obtain the statistical PDF of $\operatorname{SINR}(2, n)$. We can see that the curve for (27) and the statistical result for (14) are consistent with each other, indicating the correctness of the above derivation.

\section{Appendix B}

$$
\begin{gathered}
\int_{-\infty}^{\theta} f_{2}(s) d s=\int_{0}^{\theta} \frac{2 s\left(\theta+\delta_{1}\right)^{2}}{\left[s^{2}+\left(\theta+\delta_{1}\right)^{2}\right]^{2}} d s=\left.\left(\theta+\delta_{1}\right)^{2}\left[-\frac{1}{s^{2}+\left(\theta+\delta_{1}\right)^{2}}\right]\right|_{0} ^{\theta}=\frac{\theta^{2}}{\theta^{2}+\left(\theta+\delta_{1}\right)^{2}} . \\
\int_{-\infty}^{+\infty} f_{2}(s) d s=\int_{0}^{+\infty} \frac{2 s\left(\theta+\delta_{1}\right)^{2}}{\left[s^{2}+\left(\theta+\delta_{1}\right)^{2}\right]^{2}} d s=\left.\left(\theta+\delta_{1}\right)^{2}\left[-\frac{1}{s^{2}+\left(\theta+\delta_{1}\right)^{2}}\right]\right|_{0} ^{+\infty}=1 .
\end{gathered}
$$

Therefore, we have

$$
\frac{\int_{-\infty}^{\theta} f_{2}(s) d s}{\int_{-\infty}^{+\infty} f_{2}(s) d s}=\frac{\theta^{2}}{\theta^{2}+\left(\theta+\delta_{1}\right)^{2}}=\alpha .
$$

Hence,

$$
\delta_{1}=(\sqrt{1 / \alpha-1}-1) \theta
$$

\section{Appendix C}

Let $g=\left(\theta+\delta_{1}\right) x+y$, where $x$ and $y$ obey the i.i.d. Rayleigh distribution, we thus obtain

$$
\left\{\begin{array}{l}
x=\frac{g-y}{\theta+\delta_{1}} \\
y=y
\end{array}\right.
$$


The Jacobian determinants can be calculated as

$$
J=\left|\begin{array}{ll}
\frac{\partial x}{\partial y} & \frac{\partial x}{\partial s} \\
\frac{\partial y}{\partial y} & \frac{\partial y}{\partial s}
\end{array}\right|=\left|\begin{array}{cc}
\frac{-1}{\theta+\delta_{1}} & \frac{1}{\theta+\delta_{1}} \\
1 & 0
\end{array}\right|=-\frac{1}{\theta+\delta_{1}} .
$$

Then, the joint PDF of $y$ and $g$ can be written as

$$
f(y, g)=f(x, y)|J|=\frac{(g-y) y}{\left(\theta+\delta_{1}\right)^{2} \sigma^{4}} e^{-\frac{(g-y)^{2}+\left(\theta+\delta_{1}\right)^{2} y^{2}}{2 \sigma^{2}\left(\theta+\delta_{1}\right)^{2}}} .
$$

Let $c=\left(\theta+\delta_{1}\right)^{2}$, (34) can be simplified as

$$
f(y, g)=\frac{g y-y^{2}}{c \sigma^{4}} e^{-\frac{(g-y)^{2}+c y^{2}}{2 c \sigma^{2}}}=\frac{e^{-\frac{g^{2}}{2(c+1) \sigma^{2}}}}{c \sigma^{4}}\left(g y-y^{2}\right) e^{-\frac{(c+1)\left(y-\frac{g}{c+1}\right)^{2}}{2 c \sigma^{2}}} .
$$

Therefore, the PDF of $g$ can be calculated by

$$
f_{g}(g)=\int_{-\infty}^{+\infty} f(y, g) d y=\frac{e^{-\frac{g^{2}}{2(c+1) \sigma^{2}}}}{c \sigma^{4}} \int_{0}^{g}\left(g y-y^{2}\right) e^{-\frac{(c+1)\left(y-\frac{g}{c+1}\right)^{2}}{2 c \sigma^{2}}} d y .
$$

Let $t=\left(y-\frac{g}{c+1}\right) / \sigma$, we then have $y=\sigma t+\frac{g}{c+1}$ and $d y=\sigma d t$, and (36) can be rewritten as

$$
\begin{aligned}
f_{g}(g)= & -\frac{e^{-\frac{g^{2}}{2(c+1) \sigma^{2}}}}{c \sigma} \int_{\frac{-g}{(c+1) \sigma}}^{\frac{c g}{(c+1) \sigma}} t^{2} e^{\frac{-(c+1) t^{2}}{2 c}} d t \\
& +\frac{(c-1) g}{c(c+1) \sigma^{2}} e^{-\frac{g^{2}}{2(c+1) \sigma^{2}}} \int_{\frac{-g}{(c+1) \sigma}}^{\frac{c g}{(c+1) \sigma}} t e^{\frac{-(c+1) t^{2}}{2 c}} d t+\frac{g^{2} e^{-\frac{g^{2}}{2(c+1) \sigma^{2}}}}{(c+1)^{2} \sigma^{3}} \int_{\frac{-g}{(c+1) \sigma}}^{\frac{c g}{(c+1) \sigma}} e^{\frac{-(c+1) t^{2}}{2 c}} d t .
\end{aligned}
$$

For the first portion of (37), using integration by parts, we obtain

$$
\begin{gathered}
\int_{\frac{-g}{(c+1) \sigma}}^{\frac{c g}{(c+1) \sigma}} t^{2} e^{\frac{-(c+1) t^{2}}{2 c}} d t=\int_{\frac{-g}{(c+1) \sigma}}^{\frac{c g}{(c+1) \sigma}}\left(-\frac{c}{c+1}\right) t d\left(e^{\frac{-(c+1) t^{2}}{2 c}}\right) \\
=-\frac{c t}{c+1} e^{\left.\frac{-(c+1) t^{2}}{2 c}\right|_{\frac{-g}{(c+1) \sigma}} ^{\frac{c g}{(c+1) \sigma}}-\int_{\frac{-g}{(c+1) \sigma}}^{\frac{c g}{(c+1) \sigma}} e^{\frac{-(c+1) t^{2}}{2 c}} d t} \\
=-\frac{c^{2} g}{(c+1)^{2} \sigma} e^{\frac{-c g^{2}}{2(c+1) \sigma^{2}}}-\frac{c g}{(c+1)^{2} \sigma} e^{\frac{-g^{2}}{2 c(c+1) \sigma^{2}}}+\frac{c}{(c+1)} \int_{\frac{-g}{(c+1) \sigma}}^{\frac{c g}{(c+1) \sigma}} e^{\frac{-(c+1) t^{2}}{2 c}} d t .
\end{gathered}
$$

For the second portion of (37), using integration by parts, we obtain 


$$
\int_{\frac{-g}{(c+1) \sigma}}^{\frac{c g}{(c+1) \sigma}} t e^{\frac{-(c+1) t^{2}}{2 c}} d t=\int_{\frac{-g}{(c+1) \sigma}}^{\frac{c g}{(c+1) \sigma}}\left(-\frac{c}{c+1}\right) d\left(e^{\frac{-(c+1) t^{2}}{2 c}}\right)=-\frac{c}{c+1}\left[e^{\frac{-c g^{2}}{2(c+1) \sigma^{2}}}-e^{\frac{-g^{2}}{2 c(c+1) \sigma^{2}}}\right] .
$$

Combining (38) and (39) with (37),

$$
\begin{aligned}
f_{g}(g)=\frac{g}{(c+1)^{2} \sigma^{2}} e^{-\frac{g^{2}}{2 \sigma^{2}}}+\frac{c g}{(c+1)^{2} \sigma^{2}} e^{-\frac{g^{2}}{2 c \sigma^{2}}} \\
+\left[\frac{g^{2}}{(c+1)^{2} \sigma^{3}}-\frac{1}{(c+1) \sigma}\right] e^{-\frac{g^{2}}{2(c+1) \sigma^{2}}} \int_{\frac{-g}{(c+1) \sigma}}^{\frac{c g}{(c+1) \sigma}} e^{\frac{-(c+1) t^{2}}{2 c}} d t .
\end{aligned}
$$

The third portion of (40) can be calculated as

$$
\begin{aligned}
\int_{\frac{-g}{(c+1) \sigma}}^{\frac{c g}{(c+1) \sigma}} e^{\frac{-(c+1) t^{2}}{2 c}} d t & =\int_{0}^{\frac{c g}{(c+1) \sigma}} e^{\frac{-(c+1) t^{2}}{2 c}} d t+\int_{\frac{-g}{(c+1) \sigma}}^{0} e^{\frac{-(c+1) t^{2}}{2 c}} d t \\
= & \frac{\sqrt{\pi c}}{\sqrt{2+2 c}} \operatorname{erf}\left(\frac{c g}{\sigma} \sqrt{\frac{1}{2 c(c+1)}}\right)+\frac{\sqrt{\pi c}}{\sqrt{2+2 c}} \operatorname{erf}\left(\frac{g}{\sigma} \sqrt{\frac{1}{2 c(c+1)}}\right) .
\end{aligned}
$$

Finally, substituting (41) into (40), we get (19). We draw the curve of (18) and the statistical result of (19) together, with $\theta=1.1, \delta_{1}=9.85$, and $\sigma=1$, as shown in Fig. 14. They are perfectly consistent with each other, indicating the correctness of the above derivation.

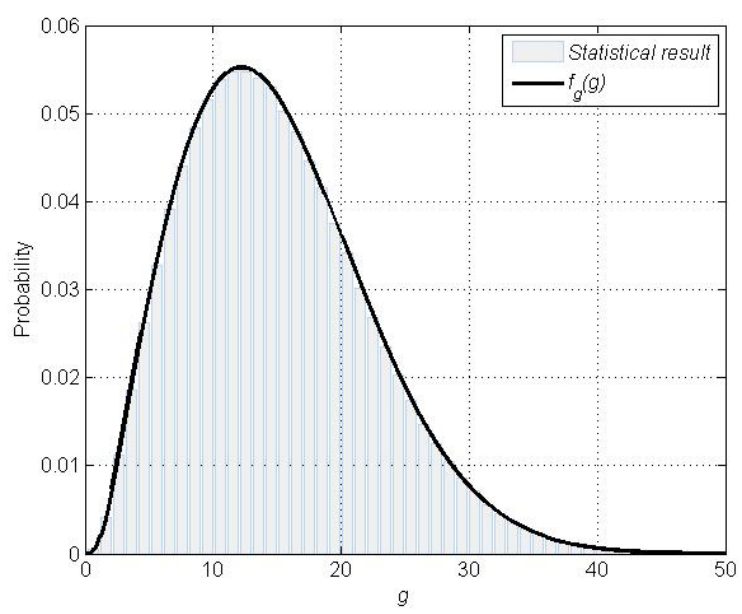

Fig. 14. Verification of the PDF derived in Appendix C.

\section{References}

[1] N. Kumaratharan and P. Dananjayan, "Performance improvement in detection and estimation of MC-CDMA systems over MIMO channels," Computers and Electrical Engineering, vol. 36, no. 1, pp. 224-233, Jan. 2010. Article (CrossRef Link).

[2] J. G. Andrews, "Interference cancellation for cellular systems: a contemporary overview," IEEE Wireless Commun., vol. 12, no. 2, pp. 19-29, April 2005. Article (CrossRef Link).

[3] J. Zhang, X. Yang, Q. Yao, X. Ge, M. Jo, and G. Mao, "Cooperative energy efficiency modeling and performance analysis in co-channel interference cellular networks," The Computer Journal, 
vol. 56, no.8, pp. 1010-1019, Aug. 2013. Article (CrossRef Link).

[4] D. T. Ngo, L. B. Le, T. Le-Ngoc, E. Hossain, and D. I. Kim, "Distributed interference management in two-tier CDMA femtocell networks,” IEEE Trans. Wireless Commun., vol. 11, no. 3, pp. 979-989, March 2012. Article (CrossRef Link).

[5] C. Jiang, Y. Shi, Y. T. Hou, W. Lou, S. Kompella, and S. F. Midkiff, "Squeezing the most out of interference: an optimization framework for joint interference exploitation and avoidance,” in Proc. of IEEE InfoCom, pp. 424-432, March 2012. Article (CrossRef Link).

[6] D. Tse and P. Viswanath, Fundamentals of Wireless Communication, Chapter 6, Cambridge Univ. Press, Sept. 2004. Article (CrossRef Link).

[7] N. I. Miridakis and D. D. Vergados, "A survey on the successive interference cancellation performance for single-antenna and multiple-antenna OFDM systems," IEEE Commun. Surv. Tut. vol. 15, no. 1, pp. 312-315, Feb. 2013. Article (CrossRef Link).

[8] D. Warrier and U. Madhow, "On the capacity of cellular CDMA with successive decoding and controlled power disparities,” in Proc. of IEEE VTC Spring, pp. 1873-1877, May 1998. Article (CrossRef Link).

[9] R. Yim, N. B. Mehta, A. F. Molisch, and J. Zhang, "Dual power multiple access with multipacket reception using local CSI,” IEEE Trans. Wireless Commun., vol. 8, no. 8, pp. 4078-4088, Aug. 2009. Article (CrossRef Link).

[10] D. Hu and S. Mao, "Multicast in femtocell networks: a successive interference cancellation approach,” in Proc. of IEEE GlobeCom, pp. 1-6, Dec. 2011. Article (CrossRef Link).

[11] R. Fa and R. C. de Lamare, "Multi-branch successive interference cancellation for MIMO spatial multiplexing systems: design, analysis and adaptive implementation,” IET Commun., vol. 5, no. 4, pp. 484-494, March 2011. Article (CrossRef Link).

[12] Lie-Liang Yang, "Receiver multiuser diversity aided multi-stage minimum mean-square error detection for heavily loaded DS-CDMA and SDMA systems,” IEEE Trans. Commun., vol. 58, no. 12, pp. 3397-3404, Dec. 2010. Article (CrossRef Link).

[13] T. Cover, “Broadcast channels,” IEEE Trans. Inf. Theory, vol. 18, no. 1, pp. 2-14, Jan. 1972. Article (CrossRef Link).

[14] M. Wildemeersch, T. Q. S. Quek, M. Kountouris, A. Rabbachin, and C. H. Slump, "Successive interference cancellation in heterogeneous networks,” IEEE Trans. Commun., vol. 62, no. 12, pp. 4440-4453, Dec. 2014. Article (CrossRef Link).

[15] S. Sasipriya and C. S. Ravichandran, "Performance analysis of overloaded CDMA system under imperfect synchronization using parallel/successive interference cancellation,” Telecom. Sys., vol. 56, no. 4, pp. 509-518, Aug. 2014. Article (CrossRef Link).

[16] Y. Shi, Y. T. Hou, J. Liu, and S. Kompella, "How to correctly use the protocol interference model for multi-hop wireless networks,” in Proc. of ACM MobiHoc, pp. 239-248, May 2009. Article (CrossRef Link).

[17] Q. Long, J. He, and C. Assi, "Understanding the benefits of successive interference cancellation in multi-rate multi-hop wireless networks,” IEEE Trans. Commun., vol. 62, no. 7, pp. 2465-2477, July 2014. Article (CrossRef Link).

[18] A. Zanella and M. Zorzi, "Theoretical analysis of the capture probability in wireless systems with multiple packet reception capabilities,” IEEE Trans. Commun., vol. 60, no. 4, pp. 1058-1071, April 2012. Article (CrossRef Link).

[19] X. Zhang and M. Haenggi, "The performance of successive interference cancellation in random wireless networks,” IEEE Trans. Inf. Theory, vol. 60, no. 10, pp. 6368-6388, Oct. 2014. Article (CrossRef Link).

[20] T. S. Rappaport, Wireless Communications: Principles and Practice, Chapter 3, 2nd Edition, Prentice-Hall, Englewood Cliffs, NJ, 2001. Article (CrossRef Link).

[21] B. Sklar, "Rayleigh fading channels in mobile digital communication systems characterization," IEEE Commun. Mag., vol. 35, no. 7, pp. 90-100, 1997. Article (CrossRef Link).

[22] Q. T. Nguyen-Vuong, Y. Ghamri-Doudane, and N. Agoulmine, "On utility models for access network selection in wireless heterogeneous networks," in Proc. of IEEE Network Operations and Manage. Symp. (NOMS), pp. 144-151, April 2008. Article (CrossRef Link). 
[23] L. Wang and G. S. Kuo, "Mathematical modeling for network selection in heterogeneous wireless networks - a tutorial,” IEEE Commun. Surv. Tut., vol. 15, no. 1, pp. 271-293, First Quarter 2013. Article (CrossRef Link).

[24] IEEE Standard, Telecommunications and information exchange between systems local and metropolitan area networks specific requirements part 11: wireless LAN medium access control (MAC) and physical layer (PHY) specifications amendment 5: television white spaces (TVWS) operation. IEEE Computer Society, 2013. Article (CrossRef Link).

[25] L. Wang, C. Goursaud, N. Nikaein, L. Cottatellucci, and J.-M. Gorce, "Cooperative scheduling for coexisting body area networks,” IEEE Trans. Wireless Commun., vol. 12, no. 1, pp. 123-133, January 2013. Article (CrossRef Link).

[26] IEEE Standard, Local and metropolitan area networks part 15.6: wireless body area networks. IEEE Computer Society, 2012. Article (CrossRef Link).

[27] IEEE Standard, Telecommunications and information exchange between systems local and metropolitan area networks specific requirements part 11: wireless LAN medium access control (MAC) and physical layer (PHY) specifications amendment 6: wireless access in vehicular environments. IEEE Computer Society, 2010. Article (CrossRef Link)

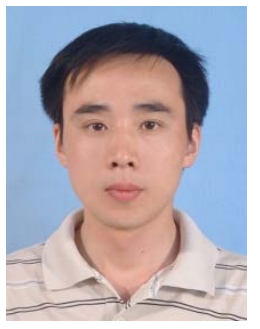

Liaoyuan Wu received his B.S. degree in Computer Technology in 1995 from North China Electric Power University (NCEPU), China and his M.S. degree in Technology of Computer Application in 2007 from Hefei University of Technology (HFUT), China. He is currently a director of technology transfer and pursuing Ph.D. degree in Hefei University of Technology. His main research interest is design and optimization of wireless network algorithm and streaming technologies.

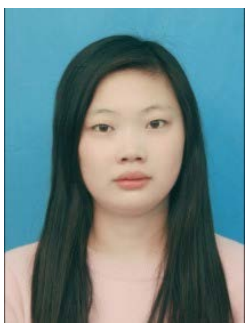

Yamei Wang received her B.Sc. degree in Communication Engineering from Huaibei Normal University in 2014. Currently, she is pursuing the M.Sc. degree at Hefei University of Technology. Her research interests include power allocation, interference coordination, and cell selection in 4G/5G networks.

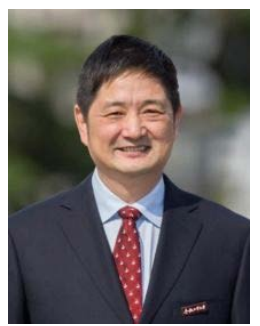

Jianghong Han received his B.S. degree in 1982 and M.S. degree in 1987 from Hefei University of Technology, China. He is now a professor and a research lab leader in School of Computer and Information, Hefei University of Technology. His research interests include computer networks, wireless sensor networks, discrete-time control systems, embedded systems and smart homes. 


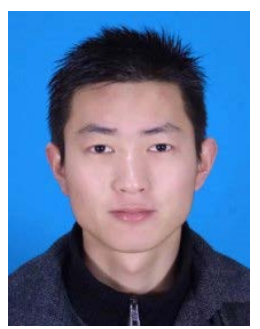

Wenqiang Chen received his B.Sc. degree in Electronic and Information Engineering from Hefei University of Technology in 2014. Currently, he is pursuing the M.Sc. degree at Hefei University of Technology. His research interests include interference coordination, resource allocation, and cell range expansion for massive-terminal IoT scenarios.

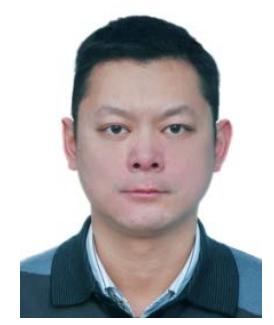

Lusheng Wang received his B.Sc. degree in Communications Engineering in 2004 from Beijing University of Posts and Telecommunications (BUPT), China and his Ph.D. degree in 2010 in Computer Science and Networks from Telecom ParisTech (ENST), France. He worked as a Post-doctoral member during 2010 in the Centre of Innovation in Telecommunications and Integration of services (CITI) at INSA-Lyon, France, and as a Post-doctoral fellow during 2011-2012 in the Department of Mobile Communications at Eurecom, Sophia Antipolis, France. Currently, he serves as a professor at Hefei University of Technology (HFUT), China. His research interests include resource and interference management in heterogeneous cellular networks and Internet of things. He has published over 30 refereed international journal and conference papers. He serves as TPC member for over 10 international conferences and as reviewer for over 10 reputed international journals. 